\title{
Staying alive
}

What are the genes essential for mammalian life? Answering such a large question requires big efforts and major bandwidth for analyzing phenotype data. Using high-throughput technologies and a large collaboration of experts and resources, researchers now have an estimate of the percentage of genes in the mouse genome necessary for development and survival (Nature 537, 508-514; 2016).

The study, supported by senior and corresponding author Stephen Murray at Jackson Laboratories (Bar Harbor, ME), involved colleagues from several institutions and the International Mouse Phenotyping Consortium (IMPC). Using 1,751 knockout mice-the first set of the nearly 5,000 knockout mice the IMPC plans to generate-and a standardized phenotyping pipeline including high-resolution 3-D imaging of whole embryos, the group identified 410 genes that were necessary for proper development and survival. Their systematic approach, combined with their imaging platform, allowed the group to observe and document phenotypes during early development on very fine scale, and uncover a remarkable amount of variability across embryos with the same mutation.

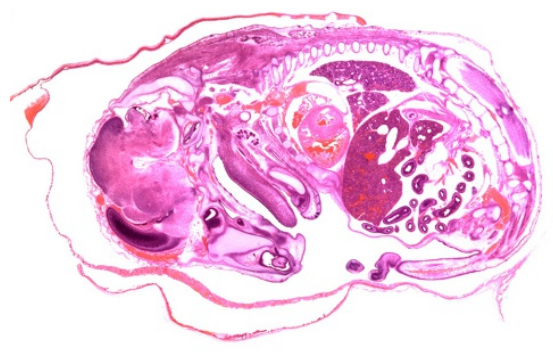

takes advantage of scale to maximize efficiency and standardize processes across multiple research centers. Another distinct quality of the study is that the large amount of data generated is completely open to the research community, which could aid scientists in academic and industry sectors. "This freely available and accessible dataset provides significant new gene-phenotype associations to enable scientists to prioritize gene candidates identified in their preclinical and discovery research," says co-first author Ann Flenniken, manager of the Clinical Phenotyping Core at The Centre for Phenogenomics (Toronto, Canada).

In addition to the data, the genetically engineered mice underlying the work are also available to the community, which should help generate more scientific returns on IMPC's investment. "This paper is just the tip of the iceberg," says co-first author Mary Dickinson of the Baylor College of Medicine (Houston, TX). "We want the scientific community to know even more about IMPC efforts and that they have access to the mice as well as the phenotype data.

Dustin M. Graham

\section{GENERALIZING GENOTYPE-PHENOTYPE RELATIONSHIPS}

For several decades, inbred mice have been used to understand the genetic underpinnings of physiology and disease. Because inbred mice have identical genetic backgrounds, manipulating a specific gene can lead to clean conclusions on its role in biological processes-or so the thinking goes. Likewise, because identical inbred lines can be maintained across institutions, results from one lab should be easily reproduced in the next lab. But what happens when researchers want to move beyond mice, and translate their findings to humans? To get at this point, Abraham Palmer and colleagues from University of California (San Diego, CA) and University of Chicago (Chicago, IL) asked an even more fundamental question: what happens when researchers try to translate findings from one mouse line to another?

Using a clever experimental design, Abraham's group created 30 lines of mutant mice with null mutations for Cacna1c or Tcf7l2, genes with known disease associations and previously established behavioral/physiological effects in mice (Neuron 91, 1253-1259; 2016). C57BL/6] males heterozygous for either Cacna1c or $T c f 7 / 2$ null mutation were crossed with wild-type females from 30 different inbred strains, generating distinct lines of F1 progeny with siblings differing only at the targeted allele. Because their modified mouse lines had the same gene mutated (either Cacna1c or $T c f 7(2)$, but also had different genetic backgrounds, the research team could directly test how well the genotype-phenotype relationships generalized across mouse lines.

For both Cacna1c and Tcf7l2-null lines, Abraham's group found strong interactions between phenotype and genetic background, and for some phenotypes the conclusions drawn from mutant mice of one line were the exact opposite of those found in another line. The results have significant implications for basic and preclinical research, which relies heavily on genetically modified mice. While Abraham's work doesn't invalidate the clear contributions that transgenic mice have made to biology and medicine, it is a tale of caution to researchers wishing to extrapolate data from rodents to humans: the results might not even apply to the mice next door. 